\title{
Futuros Profesores de Matemáticas: Rol de la Disponibilidad Léxica, Esquemas de Razonamiento Formal en Logros Académicos Durante su Formación Inicial
}

\author{
Gamal A. Cerda ${ }^{(1)}$, Pedro A. Salcedo(1), Carlos E. Pérez ${ }^{(2)}$ y Verónica Marín ${ }^{(3)}$ \\ (1) Departamento de Metodología de la Investigación e Informática Educativa, Universidad de Concepción, \\ Casilla 160-C, Concepción, Chile. (e-mail: gamal.cerda@udec.cl; psalcedo@udec.cl) \\ (2) Departamento de Ingeniería Matemática, Universidad de Concepción. (e-mail: carlos.perez@udec.cl) \\ (3) Departamento de Educación, Universidad de Córdoba, España. (e-mail: ed1madiv@uco.es)
}

Recibido May. 16, 2016; Aceptado Jun. 29, 2016; Versión final Jul. 25, 2016, Publicado Feb. 2017

\begin{abstract}
Resumen
El presente trabajo reporta los resultados de un estudio de predictibilidad de variables socio educativas y cognitivas sobre el rendimiento académico de estudiantes de la carrera de Pedagogía en Matemáticas (muestra de 173 individuos). Se examina cómo la variabilidad del promedio de las calificaciones de los estudiantes que han aprobado en el área de matemáticas durante su formación inicial puede ser explicada mediante el análisis de sus niveles de inteligencia lógica, léxico disponible y el nivel de desarrollo de esquemas de razonamiento formal, además del género y el nivel educativo. Mediante un diseño de carácter descriptivo correlacional y un análisis de regresión lineal se pudo comprobar que un porcentaje importante de la varianza de las calificaciones puede ser explicada por los modelos presentados. De especial relevancia es el léxico disponible en los estudiantes y los esquemas de razonamiento lógico formal. Se discute la necesidad de fortalecer el desarrollo de dichas habilidades en los procesos de formación inicial de matemáticas.
\end{abstract}

Palabras clave: formación inicial; profesores de matemática; léxico disponible; razonamiento formal; rendimiento académico

\section{Future Mathematics Teachers: the Role of Lexical Availability, Formal Reasoning Schema on their Academic Achievements during the Initial Formation and Training}

\begin{abstract}
This work presents the results of a study about the predictability of socio-educational and cognitive variables affecting academic performance of future mathematics teachers (sample of 173 persons). The study first analyses how variability in future mathematics teachers' GPA scores during their university training is explained by means of analyzing their levels of logical intelligence, available lexicon, the level of development of their formal reasoning schema, gender and educational level. A correlational descriptive design and a linear regression analysis were used for this and it was confirmed that a significant percentage of the variance of future mathematics teachers' GPA scores is explained by means of the models presented in this article. Specifically, both available lexicon and formal reasoning schema, show to be particularly relevant in these models. The need to strengthen the development of these abilities in pre-service mathematics teacher training in Chile is finally discussed.
\end{abstract}

Keywords: pre-service teacher training; mathematics teaching education; available lexicon; formal reasoning; academic performance 


\section{INTRODUCCIÓN}

El sistema de Educación Superior en Chile ha tenido un crecimiento exponencial en las últimas décadas, al punto que siete de cada diez estudiantes son primera generación en la universidad (SIES, 2012). En paralelo, con este aumento en la matrícula se incrementó de forma significativa el número de estudiantes que fracasan o desertan, evidenciado en el aumento del promedio de años para egresar o en el índice de titulación insatisfactoria (MINEDUC, 2012a; Centro de Microdatos, 2008). Esta extensión en el plazo de los años necesarios para titularse como también en las tasas de abandono, generan pérdidas relevantes al desarrollo del país, como también afectan especialmente a los grupos más desfavorecidos que han logrado su ingreso a la educación superior, especialmente por la magnitud del fenómeno e implicaciones sociales y económicas (Donoso y Schiefelbein, 2007; Casanova, 2015).

Por su parte, las universidades deben hacer esfuerzos adicionales en sus procesos de formación inicial para contribuir a disminuir esta realidad, implementando programas remediales y mejorando sus procesos de diagnóstico de competencias para la vida universitaria, que permitan el acceso de estudiantes que presentan las condiciones, aptitudes y actitudes adecuadas para enfrentarse a las exigencias académicas (García, 2012; Pérez et al, 2013). En el caso específico de la formación de profesores de matemáticas, tanto el fracaso como los bajos logros no son de modo alguno ajenos, así lo evidencian las evaluaciones de TEDS-M (Teacher Education Study in Mathematics) que evalúa la formación inicial docente en matemáticas para educación primaria y secundaria, ubicando a Chile en el lugar 15 entre 16 países participantes (Tatto et al., 2012). A partir de ello, resulta imperioso disponer de diagnósticos que arrojen luces sobre posibles variables asociadas al desempeño de los estudiantes a lo largo de todo su proceso de formación, con la intención de poder implementar de forma temprana aquellos mecanismos remediales o actividades que vayan en el logro a través del refuerzo de estas habilidades, sobre todo teniendo en cuenta que, en general, las instituciones y procesos de formación han continuado desarrollando prácticas académicas que no consideran adaptaciones pertinentes a la nueva población estudiantil, dado que en ellas existe una idea ampliamente extendida en las comunidades universitarias, de que un estudiante universitario se encuentra perfectamente capacitado para enfrentar sus estudios con éxito dependiendo exclusivamente de su esfuerzo (Leese, 2010; Tuckman y Kennedy, 2011). Esta idea desvincula a la universidad como responsable de la provisión de recursos adecuados para que el alumno alcance los aprendizajes esperados, pues en general, se introduce al estudiante, sin ningún periodo de aclimatación, en un sistema en el que se le exige que asuma la responsabilidad de su propio aprendizaje, gestione su tiempo y recursos para adquirir una formación acorde a las nuevas demandas sociales y profesionales (Romero y Pérez, 2009).

La detección temprana de factores que inciden en el rendimiento académico mediante el examen de este tipo de variables asociadas al estudiante permite ofrecer un contexto de apoyo institucional en la propia formación, evitando además estigmatizar al estudiante como único responsable, al asumir que el estudiante de nivel superior se encuentra perfectamente capacitado para enfrentar sus desafíos de aprendizaje con éxito dependiendo exclusivamente de su esfuerzo (Sanford y Hunter, 2011). El desempeño académico del estudiante universitario parece ser un complejo proceso en el que interviene un conjunto de variables asociadas al contexto sociocultural del alumno como también a variables de tipo transversal asociadas al aprendizaje (Yau et al., 2012; Salazar y Leihey, 2013). Adicionalmente a los requerimientos académicos, los estudiantes, especialmente aquellos de primeros años, han de sumar las dificultades inherentes al proceso de transición de la educación secundaria hacia un nuevo mundo social y educativo (Kostons et al., 2012).

Por todo lo anterior, se hace necesario desarrollar investigaciones para determinar aquellos factores 0 variables de tipo cognitivo o sociodemográficas, que pueden desempeñar un rol importante a la hora de explicar el logro o presencia de dificultades en el ámbito de las matemáticas. Dentro de las variables de tipo cognitivo asociadas al rendimiento en matemáticas se encuentra la inteligencia lógica. Al respecto, se puede mencionar para el caso de estudiantes chilenos de educación preuniversitaria, la existencia de investigaciones que reportan su relación positiva y significativa tanto con el rendimiento en matemáticas como también con el rendimiento académico en general (Cerda et al., 2011), pero no se ha analizado su relación con alumnado en nivel universitario. El nivel de desarrollo de los esquemas de razonamiento formal de los estudiantes se relaciona con su rendimiento en matemáticas, dado que dichos alumnos son capaces de poner en juego habilidades de visualización, como para aumentar o disminuir mentalmente los tamaños de objetos, situar espacialmente los objetos, tener mejores niveles de memoria de formas y habilidad para manipular objetos tridimensionales, esto se ha constatado en estudiantes de ingeniería (Jones et al., 2011; Maris y Difabio, 2009).

Por otra parte, en matemáticas es fundamental el uso de una serie de conceptos y términos disciplinares que resultan fundamentales para una correcta apropiación de los aprendizajes en dicho ámbito, y por ende, resulta crucial determinar el vocabulario de los estudiantes asociado a distintos temas o ejes matemáticos 
fundamentales. Esta especie de glosario disciplinar se puede asimilar a la "palabra disponible", que es aquella que, sin ser frecuente, se presenta en forma inmediata y natural a la mente, justo en el momento en que el individuo la necesita. Este léxico potencial pertenece al número de palabras de uso activo por parte del individuo y está propenso a la actualización en la comunicación cotidiana (Urzúa et al., 2006). Hay evidencia de que los alumnos no tienen un léxico disponible adecuado, presentan deficiencias para el desarrollo de estructuras semánticas que les permitan avanzar en forma óptima en la adquisición del conocimiento, especialmente en el ámbito de las matemáticas (Ferreira et al., 2014; Salcedo et al., 2013). No obstante, a juicio de los autores, no hay estudios en Chile que vinculen dichos niveles de disponibilidad léxica con el rendimiento académico específico en las asignaturas relacionadas con matemáticas en educación superior.

En este sentido, el estudio de diversas variables o factores asociados al rendimiento escolar en matemáticas en estudiantes de Pedagogía en Matemática, pueden contribuir de forma significativa en los procesos de mejora de las prácticas pedagógicas de ellos como futuros docentes o de aquellos que actualmente están en pleno proceso de formación. Examinar estos factores y variables vinculadas con el razonamiento matemático y la disponibilidad léxica en estudiantes universitarios de pedagogía en matemáticas, cobra particular importancia, cuando se tiene a la luz que los estudiantes que egresan del ciclo de formación secundaria en matemáticas, deberían ser capaces enfrentar de forma satisfactoria situaciones didácticas o problemas en los que para poder resolverlos, utilicen distintos registros de representación junto a un discurso matemático significativo y sean capaces de usar representaciones matemáticas y procedimentales desde la comprensión conceptual, entre otras (NCTM, 2000). Para ello, se debiera disponer de mediciones válidas y confiables de las variables anteriormente mencionadas, posibilitando allegar información fundamental al sistema educativo, en especial a los centros de formación inicial de profesores de matemáticas, que les permita planificar y gestionar de mejor forma los procesos de enseñanza-aprendizaje orientados al logro de aprendizajes significativos, impactando directa e indirectamente en los índices de disminución del fracaso y abandono, y pudiendo contribuir por cierto a una mejora significativa de los bajos resultados obtenidos en las pruebas INICIA de conocimientos disciplinares de matemáticas que realizan los profesores recién egresados de las instituciones de educación superior (MINEDUC, 2012b).

La investigación presente abordar las siguientes preguntas de investigación: (1)¿Cómo se relaciona el número de palabras o léxico disponible asociados a los ejes de Sistemas numéricos, Cálculo, Estructuras, Geometría y Datos y Azar; el nivel de desarrollo y tipo de esquemas de razonamiento formal y la inteligencia lógica con el rendimiento académico de los futuros profesores de matemática?; (2) ¿Es posible encontrar un modelo predictivo que incorpore todas las variables anteriormente señaladas, e idealmente otras variables como la institución donde asisten, género y nivel educativo en el cual se encuentran dichos estudiantes, que permita explicar la variabilidad de sus promedios de calificaciones obtenidas en asignaturas del área de formación de la especialidad?. El objetivo principal de la investigación postula determinar el rol de diversas variables de tipo cognitivas y socio-educativas, respecto del logro académico de los futuros profesores de matemáticas durante el desarrollo de sus estudios que permita contribuir a los procesos de formación inicial de los profesores de dos instituciones de educación superior en Chile.

\section{MÉTODOLOGÍA}

El estudio es de tipo descriptivo y correlacional. El diseño es ex post facto, debido a que las variables ya tomaron sus valores al momento de ser medidas. Dado que el objetivo postula examinar el grado de predictibilidad de una serie de variables sobre el rendimiento académico de los estudiantes de pedagogía en matemáticas en asignaturas de carácter disciplinar.

\section{Participantes}

La muestra estuvo constituida por 173 estudiantes de Pedagogía en Matemáticas de primer a quinto año de las Carreras de Pedagogía en Matemática impartidas en dos universidades $(57,8 \%$ y 42,2\% respectivamente). La mayor proporción de estudiantes corresponde a primer y segundo año (30,6\% y 24,9\% de total respectivamente). El 53,2 \% de los estudiantes es de género masculino y 46,8\% femenino. Estas dos variables también se incorporaron al estudio. La elección de dos universidades, se realizó en función que ambas tienen procesos de formación inicial de profesores de matemáticas, y se encuentran en la misma ciudad. Además, ambas instituciones tienen acreditadas todas las carreras de Pedagogía que imparten, y son similares en su estructura y número de facultades. Una de ellas es de carácter público, y la otra siendo privada, es de carácter tradicional y pertenece al Consejo de Rectores de las Universidades Chilenas, $\mathrm{CRUCH}$. Además, ambas instituciones universitarias atienden una población estudiantil de un nivel socioeconómico similar. 


\section{Variables e Instrumentos}

Inteligencia lógica: Esta variable se midió a través del Test de Inteligencia Lógica Superior (TILS) (Cerda et al., 2010). Es la capacidad que tienen las personas para vislumbrar soluciones y resolver problemas, estructurar elementos para realizar deducciones y fundamentarlas con argumentos válidos acordes con su desarrollo cognitivo de acuerdo con el enfoque piagetiano. Esta habilidad implica la capacidad para reconocer en una serie de elementos la regla general que los rige o subyace a los mismos. El constructo se operacionaliza como el puntaje que alcanza un alumno al completar un conjunto de ejercicios de series incompletas de carácter figurativo (puntaje TILS), cuya escala de puntajes es de 0 a 50 puntos. El alfa de Cronbach es ,94. Los resultados de los análisis factoriales del instrumento permitieron concluir que el modelo de un factor es el que presenta los mejores índices de ajuste: $\mathrm{X} 2(1.175)=4.784,38, p=, 001 ; \mathrm{CFI}=$ , $97 ; \mathrm{TLI}=, 97 ; \mathrm{RMSEA}=, 05$. Esto permite asumir el uso de su puntuación global en el análisis.

Esquemas de razonamiento formal: Para examinar esta variable se utilizó el Test of Logical Thinking, TOLT (Tobin y Capie, 1981). Los esquemas de razonamiento formal permiten abordar problemas de forma abstracta, formulando supuestos o hipótesis, llevando a cabo operaciones proposicionales de segundo orden: compensación, disyunción, implicación, disociación, entre otras. La prueba examina cinco esquemas de razonamiento, a saber: Proporcionalidad (PP), que examina tareas donde se opera con razones y proporciones; Control de variables $(\mathrm{CV})$, evalúa tareas o situaciones en las que exista más de un sistema variable que pueda determinar el objeto observado; Probabilidad (PB), evalúa tareas donde se poner en juego la comprensión de la relación entre azar y proporción; Correlación (CR), se evalúa tareas en que se examina la comprensión de la variación conjunta de dos o más variables, y Combinatoria (CB); consiste en combinar objetos y proposiciones de todas las maneras posibles, sirviéndose de nociones matemáticas como la combinación, permutación y variación. Las ocho primeras tareas poseen una estructura de dos niveles, es decir, se debe seleccionar tanto la respuesta como la explicación entre 5 alternativas, que han sido configuradas sobre la base de los errores sistemáticos más frecuentes en los que se suele incurrir en la resolución de este tipo de problemas. Las dos últimas tareas, referidas a permutaciones y combinatorias, son de respuesta abierta de tipo semiestructurado. Los individuos disponen de 38 minutos para responder el test. El Alfa de Cronbach, arrojó un índice $r_{\alpha}=, 95$. El análisis de su estructura factorial es adecuada y corresponden a un $\chi 2(29)=34,311, p=, 228, \mathrm{CFI}=1,00, \mathrm{TLI}=1,00$, RMSEA $=, 01 \quad(95 \%$ IC:, $00-, 03)$. También se procedió a analizar las correlaciones entre las dimensiones con la finalidad de examinar la existencia de un factor de segundo de orden, que permitiera considerar su puntuación global, y los resultados también resultan adecuados, $\chi 2(31)=38,430, p=168, \mathrm{CFI}=1,00, \mathrm{TLI}=1,00, \mathrm{RMSEA}=, 01$ (95\% IC: ,00-,03), lo cual permite concluir que efectivamente el modelo de un factor de segundo orden ajusta de manera adecuada los datos analizados.

Léxico disponible: es el número de las palabras que se presentan en la mente del estudiante de forma inmediata y natural cuando se trata un determinado tema. Se especificaron 5 centros de interés: Sistemas numéricos, Cálculo, Estructuras, Geometría y Datos y Azar. Para determinar el número de palabras asociado a cada eje de interés, se utilizó una versión de la prueba de disponibilidad léxica virtual, utilizando para ello el software LexMath (Salcedo et al., 2013). Ello permite una aplicación masiva con bajo costo, pues se aplica vía Internet, con un tiempo de controlado de dos minutos. Se utilizaron estrategias heurísticas para determinar errores ortográficos y palabras mal escritas, y almacenando todo en una base de datos con los datos de identificación de cada encuestado.

Rendimiento Académico: Promedio de calificaciones, expresado en escala de 1 a 7 , de todas las asignaturas del ámbito matemático o disciplinar que los estudiantes hayan cursado en la universidad hasta el momento de la investigación, de acuerdo al nivel o año de formación en que se encuentran.

\section{Procedimiento}

Se llevaron a cabo diversas visitas a las dos universidades que participaron del estudio, con la finalidad de identificar el número de estudiantes de cada curso desde primer a quinto año, calendarizar la aplicación de los instrumentos y el acceso a la información sobre sus calificaciones. Para todos los efectos, se contó con el consentimiento informado de los estudiantes y de las autoridades de dichas instituciones y carreras, quienes manifestaron su anuencia a participar de forma libre, y con la posibilidad de dejar de hacerlo cuando estimaran conveniente.

El análisis de resultados se realizó en dos niveles. Primero, se calculó el valor predictivo de las variables en relación con el promedio de calificaciones en las asignaturas del área de matemáticas que obtienen los estudiantes. Después, se analizaron las variables de forma comparativa, mediante la prueba $t$ y ANOVA, examinándose además las relaciones de carácter bivariado, mediante el coeficiente producto momento de Pearson. Finalmente, se aplicó una técnica de análisis multivariante de regresión lineal múltiple por pasos 
(stepwise) con el fin de establecer cómo ciertas variables consideradas tradicionalmente como predictoras o explicativas en la literatura especializada se relacionan con la variable criterio. Una vez finalizadas las evaluaciones, se procedió a realizar los análisis estadísticos mediante el programa estadístico SPSS ${ }^{\circledR}$ versión 19.

\section{RESULTADOS Y DISCUSIÓN}

La investigación persigue establecer y cuantificar el valor predictivo de las diversas variables contempladas respecto del rendimiento académico de los estudiantes en las diversas asignaturas que ellos han cursado. La distribución de la variable rendimiento académico presentó valores de asimetría y curtosis dentro del rango esperado (,367 y -,429 respectivamente). Además, se constató que la distribución de calificaciones de los estudiantes en función del género en ambos grupos es igualmente normal y hay diferencias significativas al comparar sus rendimientos medios a favor del grupo masculino $(M=4,936, D T=, 525$ y $M=4,773, D T=$ ,496 respectivamente; $(F(1,171)=4,358, p<, 05)$, además de comprobarse la homocedasticidad (prueba de Levene $=, 095 ; p>, 05)$.

\section{Variables de contraste}

Al analizar los datos respecto de variables de contraste tales como niveles de inteligencia lógica, esquemas de razonamiento, léxico disponible, en función del género, nivel educativo y universidad a la que asisten los estudiantes, se observa que hay diferencias en las puntuaciones en inteligencia lógica de los estudiantes de Pedagogía en Matemáticas al comparar estas en función de la institución universitaria a la cual asisten, $(F(1,171)=8,973, p>, 001)$, además de comprobarse la homocedasticidad (prueba de Levene $=, 077 ; p<$ ,05). También se observan diferencias significativas en las puntuaciones medias de inteligencia lógica en función del nivel educativo (Welch $F(3 ; 86,419)=4,055 p<, 01$ ), y ellas favorecen al grupo de estudiantes de segundo año respecto del grupo de estudiantes de curso superior (Games-Howel, diferencia de medias (I$J)=4,893, p<, 05)$. Sin embargo, no hay diferencias al comparar esas puntuaciones en función del género de los estudiantes.

En cuanto a los esquemas de razonamiento formal, se observan diferencias a nivel de la puntuación global obtenida en función del nivel educativo, aun cuando no es posible señalar que las varianzas de los grupos sean homogéneas (Welch $F(3 ; 89,188)=2,717, p<, 05$. La diferencia fundamental está dada por la puntuación de los estudiantes de tercer año respecto a los estudiantes de segundo año. También, hay diferencias significativas al comparar la puntuación global en función del tipo de institución a la cual asisten (Welch $F(1 ; 131,826)=11,446 p<, 001)$. Pero no se observan diferencias al comparar los grupos en función del género.

Dentro de los diversos esquemas de razonamiento, se observa que existen diferencias significativas en tres de los cinco esquemas, al comparar la actuación de los estudiantes en función de la institución a la cual asisten, a saber: Control de variable, Probabilidades y combinatoria $(F(1,171)=4,116, p<, 044 ; F(1,171)=$ $4,058, p<, 046 ; F(1,171)=14,119, p<, 001$, respectivamente). Al comparar los esquemas de razonamiento por género, sólo se observan diferencias significativas a favor del grupo masculino en el esquema de razonamiento que implica resolver tareas de probabilidad, $(F(1,171)=7,966, p<, 005)$. En cuanto al nivel educativo de los estudiantes, se observan diferencias significativas en los esquemas de razonamiento que implican resolver tareas de proporcionalidad $(F(3,169)=3,931, p<, 01$ y de correlación $(F(3,169)=2,986, p$ $<, 05)$, fundamentalmente asociadas a la diferencia de los estudiantes de cursos superiores comparados con primeros años.

En cuanto al léxico disponible o número de palabras referidas a los cinco centros de interés o temas, no se observan diferencias significativas en función del género en ninguno de ellos. Sin embargo, al compararlos por la institución en la que estudian, se observan diferencias significativas en 4 de ellos, a saber: Cálculo, estructuras, geometría y datos y azar $(F(1,171)=13,085, F(1,171)=18,652, F(1,171)=54,786, F(1,171)=$ $28,377$, todas ellas con $p<, 001)$. Finalmente, al comparar el léxico disponible en función del nivel educativo, se observan diferencias en cada uno de ellos: sistemas numéricos $(F(3,169)=6,646, p<, 001)$; cálculo $(F(3,169)=14,223, p<, 001)$; estructuras $(F(3,169)=17,092, p<, 001)$; geometría $(F(3,169)=$ $20,992, p<, 001)$ y sistemas numéricos $(F(3,169)=26,686, p<, 001)$.

\section{Análisis de correlaciones entre variables}

Existe una relación positiva y significativa entre el mayor puntaje obtenido al resolver tareas de probabilidades de los estudiantes y el promedio de calificaciones que ellos alcanzan $[r(173)=, 306$, $p<$ ,001], como también, al asociar la puntuación global alcanzada en el TOLT y las calificaciones $[r(173)=$ ,269, $p<, 001]$. En cuanto al número de palabras o léxico disponible, se observan relaciones positivas y 
significativas en cuatro de los centros de interés con el rendimiento académico, a saber: número de palabras en cálculo $[r(173)=, 369, p<, 001]$; estructura $[r(173)=, 391, p<, 001]$; geometría $[r(173)=, 481, p<, 001]$ y datos y azar $[r(173)=, 465, p<, 001]$. No se observan asociaciones significativas de la inteligencia lógica con el rendimiento académico. En cuanto a las relaciones entre las variables, se puede destacar la asociación positiva y significativa entre el número de palabras en cada centro de interés con los otros, lo que estaría sugiriendo que aquellos estudiantes que tienden a generar más palabras a un tema también tienden a producir mayor número de palabras en los otros y viceversa.

\section{Modelo de regresión lineal}

Por último, se realizó un análisis de regresión lineal por pasos. Se incorporaron todas las variables que fueron anteriormente descritas sumadas a aquellas variables como género, universidad y nivel educativo, con la finalidad de determinar un modelo suficientemente parsimonioso que explicara los pesos y porcentajes de la variabilidad del promedio de calificaciones académicas en las asignaturas de matemáticas de los estudiantes de las carreras de pedagogía en matemática. El resumen de ello, puede observarse en la Tabla 1.

Tabla 1: Modelo de regresión lineal múltiple por pasos.

\begin{tabular}{|c|c|c|c|c|c|c|c|c|}
\hline \multirow[b]{2}{*}{ Modelo } & \multirow[b]{2}{*}{$\mathrm{R}$} & \multirow[b]{2}{*}{$\mathrm{R} 2$} & \multirow{2}{*}{$\begin{array}{l}\mathrm{R} 2 \\
\text { corregida }\end{array}$} & \multirow[b]{2}{*}{$\begin{array}{l}\text { Error típ. } \\
\text { estimación }\end{array}$} & \multicolumn{3}{|c|}{ Estadísticos de cambio } & \multirow[b]{2}{*}{$\begin{array}{l}\text { Durbin } \\
\text { Watson }\end{array}$} \\
\hline & & & & & $\begin{array}{l}\text { Cambio en } \\
\text { R2 }\end{array}$ & $\begin{array}{l}\text { Cambio en } \\
\mathrm{F}\end{array}$ & $\begin{array}{l}\text { Sig. } \\
\text { Cambio } \mathrm{F}\end{array}$ & \\
\hline 1 &, $559 a$ & ,312 & ,308 & ,4293 & ,312 & 77,614 & ,000 & \\
\hline 2 & ,622b & ,387 & ,380 & ,4064 & ,075 & 20,854 &, 000 & \\
\hline 3 & ,656c & ,431 & ,420 & ,3929 & ,043 & 12,827 &, 000 & \\
\hline 4 & ,667d & ,445 & ,431 & ,3892 &, 014 & 4,254 &, 041 & \\
\hline 5 &, $677 e$ & ,459 & ,443 & ,3853 &, 014 & 4,376 & ,038 & 1,861 \\
\hline
\end{tabular}

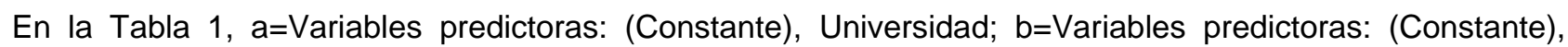
Universidad, Número de palabras datos y azar; $\mathrm{c}=$ Variables predictoras: (Constante), Universidad, Número

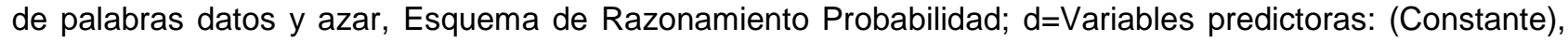
Universidad, Número de palabras datos y azar, Esquema de Razonamiento Probabilidad, Nivel Educativo; e=Variables predictoras: (Constante), Universidad, Número de palabras datos y azar, Esquema de Razonamiento Probabilidad, Nivel Educativo, Género del estudiante; $f=$ Variable a predecir: Promedio Académico Ponderado

Como se observa en la Tabla 1, del análisis de regresión surgen 5 modelos, cada uno con su propia capacidad explicativa. Para el modelo 5 , su coeficiente de correlación múltiple fue $R=, 690$ y el coeficiente de determinación $R^{2}=, 459$, que se ajustó a $R^{2}=, 443$. Por tanto, se puede inferir que el $44,3 \%$ de la varianza de las calificaciones en las asignaturas de matemáticas puede ser explicada por las siguientes seis variables: Universidad donde estudia, Número de palabras en datos y azar, Esquema de Razonamiento Probabilidad, Nivel Educativo, Género del estudiante, con un Durbin-Watson $=1,861$ considerado adecuado. Como se observó al analizar las correlaciones previas, no es sorprendente que sólo cargara en el modelo un centro de interés, debido a la asociación positiva y significativa entre el número de palabras en cada centro de interés con los otros. La aparición del esquema de razonamiento de probabilidad por sobre el resto probablemente se deba a que dicho esquema que presentaba diferencias significativas respecto de las variables género y universidad. Se observa también que el modelo 3, en un enfoque más parsimonioso, podría considerarse un modelo con solo tres variables: Universidad, Número de palabras datos y azar, Esquema de Razonamiento Probabilidad, tiene una capacidad explicativa de $42 \%$, y su valor del estadístico de Durbin-Watson $=1,839$, que puede ser igualmente considerado como adecuado. El peso relativo de las variables fue: Universidad $(\beta=-, 420)$, léxico disponible datos y azar $(\beta=, 283)$, esquema de razonamiento probabilidad $(\beta=, 211)$.

Para garantizar la validez del modelo, se analizó la independencia de los residuos. El estadístico $D$ de Durbin-Watson obtuvo un valor $\mathrm{D}=1,839$, confirmando la ausencia de autocorrelación positiva (valores cercanos a 0) y negativa (valores cercanos a 4). Del mismo modo, se asumió la ausencia de colinealidad y, por tanto, la estabilidad de las estimaciones al obtener altos valores de tolerancia y bajos valores en los factores de inflación de la varianza (FIVs). Los valores de tolerancia muy pequeños indican que esa variable puede ser explicada por una combinación lineal del resto de las variables, lo cual indicaría la existencia de colinealidad (valores cercanos a ,01), pero no es el caso. Por otro lado, los FIV son los inversos de los niveles de tolerancia, y cuanto mayor es el FIV de una variable, mayor es la varianza del correspondiente coeficiente de regresión y, por ende, habría una inestabilidad en las estimaciones, pero tampoco es el caso. 
Tabla 2: Coeficientes del modelo de regresión lineal múltiple

\begin{tabular}{|c|c|c|c|c|c|c|c|c|}
\hline \multirow{2}{*}{\multicolumn{2}{|c|}{ Modelo }} & \multicolumn{2}{|c|}{$\begin{array}{l}\text { Coeficientes } \\
\text { estandarizados }\end{array}$} & \multirow{2}{*}{$\begin{array}{c}\begin{array}{c}\text { noCoeficientes } \\
\text { tipificados }\end{array} \\
\text { Beta } \\
\end{array}$} & \multirow[b]{2}{*}{$\mathrm{T}$} & \multirow[b]{2}{*}{ Sig. } & \multicolumn{2}{|l|}{$\begin{array}{l}\text { Estadísticos } \\
\text { colinealidad }\end{array}$} \\
\hline & & $B$ & Error típ. & & & & Tolerancia & FIV \\
\hline \multirow[t]{4}{*}{3} & (Constante) & 4,881 & ,164 & & 29,799 &, 000 & & \\
\hline & Universidad &,- 437 & ,066 &,- 420 & $-6,649$ &, 000 & ,845 & 1,183 \\
\hline & $\begin{array}{l}\text { Número de palabras } \\
\text { datos y azar }\end{array}$ & ,024 & ,005 & ,283 & 4,512 &, 000 & ,855 & 1,170 \\
\hline & $\begin{array}{l}\text { Esquema } \\
\text { Razonamiento } \\
\text { Probabilidad }\end{array}$ & ,094 & ,026 & ,211 & 3,581 & ,000 & ,974 & 1,027 \\
\hline
\end{tabular}

Por otra parte, se analizó un modelo de predictibilidad del rendimiento académico en matemáticas, considerando sólo las variables asociadas a desempeños de los estudiantes. Para ello se empleó el mismo método de pasos sucesivos, que arrojó los siguientes resultados (véase Tabla 3).

Tabla 3: Modelos de regresión lineal múltiple por pasos

\begin{tabular}{|c|c|c|c|c|c|c|c|c|}
\hline \multirow[b]{2}{*}{ Modelo } & \multirow[b]{2}{*}{$\mathrm{R}$} & \multirow[b]{2}{*}{$\mathrm{R} 2$} & \multirow{2}{*}{$\begin{array}{l}\mathrm{R} 2 \\
\text { corregida }\end{array}$} & \multirow{2}{*}{$\begin{array}{l}\text { Error típ. } \\
\text { estimación }\end{array}$} & \multicolumn{3}{|c|}{ Estadísticos de cambio } & \multirow{2}{*}{$\begin{array}{l}\text { Durbin } \\
\text { Watson }\end{array}$} \\
\hline & & & & & $\begin{array}{l}\text { Cambio en } \\
\text { R2 }\end{array}$ & $\begin{array}{l}\text { Cambio en } \\
\mathrm{F}\end{array}$ & $\begin{array}{l}\text { Sig. } \\
\text { Cambio F }\end{array}$ & \\
\hline 1 & ,481a & ,231 & 227 & ,4538 & 231 & 51,466 & ,481a & \\
\hline 2 &, $542 b$ & ,294 & ,286 & ,4361 &, 063 & 15,149 &, $542 b$ & \\
\hline 3 &, $569 c$ & ,324 & ,312 & ,4280 &, 030 & 7,519 &, $569 c$ & \\
\hline 4 &, $592 d$ & ,351 & ,335 & ,4209 &, 026 & 6,800 &, $592 d$ & 1,796 \\
\hline
\end{tabular}

En la Tabla 3, a=Variables predictoras: (Constante), Número de palabras geometría; $b=$ Variables predictoras: (Constante), Número de palabras geometría, Esquema de Razonamiento Probabilidad; $\mathrm{C}=$ Variables predictoras: (Constante), Número de palabras geometría, Esquema de Razonamiento Probabilidad, Número de palabras datos y azar; d=Variables predictoras: (Constante), Número de palabras geometría, Esquema de Razonamiento Probabilidad, Número de palabras datos y azar, Número de palabras sistema numérico; y e=Variable a predecir: Promedio Académico Ponderado

Se optó por el modelo 4, pues explica un $33,5 \%$ de la variabilidad del rendimiento. Se puede observar en la Tabla 3 que las variables que presentaron un mayor peso fueron: léxico disponible en geometría $(\beta=, 336)$, esquema razonamiento formal probabilidades $(\beta=, 253)$, léxico disponible datos y azar $(\beta=, 289)$ y léxico disponible sistema numérico $(\beta=-, 186)$. El estadístico $D$ de Durbin-Watson obtuvo un valor $D=1,796$, confirmando la ausencia de algún tipo de autocorrelación. Del mismo modo, se asumió la ausencia de colinealidad y, por lo tanto, la estabilidad de las estimaciones al obtener altos valores de tolerancia y bajos valores en los factores de inflación de la varianza, tal como se puede observar en la Tabla 4.

Tabla 4: Coeficientes del modelo de regresión lineal múltiple

\begin{tabular}{|c|c|c|c|c|c|c|c|c|}
\hline \multirow{2}{*}{\multicolumn{2}{|c|}{ Modelo }} & \multicolumn{2}{|c|}{$\begin{array}{l}\text { Coeficientes } \\
\text { estandarizados }\end{array}$} & \multirow{2}{*}{$\begin{array}{c}\begin{array}{c}\text { noCoeficientes } \\
\text { tipificados }\end{array} \\
\text { Beta } \\
\end{array}$} & \multirow[b]{2}{*}{$\mathrm{T}$} & \multirow[b]{2}{*}{ Sig. } & \multicolumn{2}{|l|}{$\begin{array}{l}\text { Estadísticos } \\
\text { colinealidad }\end{array}$} \\
\hline & & $B$ & Error típ. & & & & Tolerancia & FIV \\
\hline \multirow[t]{5}{*}{4} & (Constante) & 3,979 & ,125 & & 31,892 & ,000 & & \\
\hline & $\begin{array}{l}\text { Número de palabras } \\
\text { geometría }\end{array}$ & s ,023 &, 006 & ,336 & 3,805 & ,000 & ,497 & 2,013 \\
\hline & $\begin{array}{l}\text { Esquema } \\
\text { Razonamiento } \\
\text { Probabilidad }\end{array}$ & e ,113 & ,028 & ,253 & 4,039 & ,000 & ,982 & 1,018 \\
\hline & $\begin{array}{l}\text { Número de palabras } \\
\text { datos y azar }\end{array}$ & s , ,025 & ,007 & ,289 & 3,278 & ,001 & ,498 & 2,006 \\
\hline & $\begin{array}{l}\text { Número de palabras } \\
\text { sistema numérico }\end{array}$ & s,- 018 &, 007 &,- 186 & $-2,608$ & ,010 & ,759 & 1,318 \\
\hline
\end{tabular}




\section{DISCUSION FINAL}

El objetivo e interés de analizar modelos complejos de predicción del rendimiento académico de futuros profesores de matemáticas durante su proceso de formación inicial, como el realizado en el presente estudio, abre una importante arista para retroalimentar los procesos mismos de formación inicial del profesorado en esta área, ya que contribuye con información relevante al reconocimiento o identificación de los estudiantes que se encuentran en riesgo de obtener bajos logros de aprendizaje y, por ende, eventualmente abandonar la carrera, o bien aumentar de forma significativa el número de años necesarios para finalizar su proceso de formación inicial.

Inicialmente, en este estudio se examinaron 15 variables que teóricamente podrían relacionarse con el desempeño académico de los estudiantes de Pedagogía en Matemáticas cursando cada uno de los 5 años de duración de la carrera. Los resultados muestran que uno de los modelos, que incluye todas las variables de tipo socioeducativas y de desempeño de los estudiantes, explica aproximadamente el $44,3 \%$ de la variabilidad observada en el promedio de calificaciones que obtienen los estudiantes en las asignaturas vinculadas a la especialidad que han cursado hasta el momento del análisis. No obstante, un enfoque más parsimonioso, permite optar por un modelo de sólo tres variables que explica el $42 \%$, dentro del cual destaca el rol de la institución en la cual se forman. Se recordará al efecto, que son estudiantes de dos instituciones de educación superior que imparten la carrera de Pedagogía en Matemáticas en la Región del Bío Bío. Las otras dos variables relevantes del modelo la constituyen el léxico disponible o número de palabras que el estudiante puede asociar al centro de interés matemático datos y azar y el nivel de resolución de tareas que implican esquemas de razonamiento de tipo probabilidad.

Estos hallazgos permiten inferir la relevancia que puede asumir la institucionalidad en los procesos de formación inicial, con sus sellos y perfiles de egreso, diferencia y énfasis en las mallas de formación, como también enfoques de enseñanza-aprendizaje, sin dejar de reconocer las características propias de los estudiantes que ingresan a sus aulas. Lo anterior, cobra especial importancia si se examinan los documentos oficiales del Ministerio de Educación de Chile, que relevan en igual importancia en el Currículo, tanto los Conocimientos (Ejes de Números, Álgebra, Geometría y Datos y Azar), como a las habilidades y las actitudes. De esta manera, se pretende que todos los estudiantes logren desarrollar durante su itinerario escolar las habilidades de razonamiento lógico, visualización espacial, pensamiento analítico, cálculo, modelamiento y la capacidad para resolver problemas. Conjuntamente con lo anterior, las orientaciones didácticas de la asignatura de Matemática buscan que éstos adquieran aprendizajes de vida, siendo la matemática un área poderosa de la cultura, pues, permite comprender, explicar y predecir situaciones y fenómenos del contexto (MINEDUC, 2012c).

Sin embargo, fuera de la diferencia en ambas instituciones, las variables de desempeño asociadas a dos dominios resultan comunes y particularmente importante destacar: el léxico disponible al centro de interés denominado datos y azar y, por otra parte, el desempeño observado al resolver tareas que involucran esquemas de razonamiento lógico formal relacionados con probabilidades. Este hecho es relevante, pues ambos dominios se asocian a uno de los ejes matemáticos centrales del proceso de formación en educación básica y media, el cual persigue que los estudiantes sean capaces fundamentalmente de recolectar, organizar, representar y analizar datos, como también, de realizar inferencias a partir de información de naturaleza estadística y distinguir entre los fenómenos aleatorios y los deterministas. De hecho, dentro de los cambios realizados a los Objetivos Fundamentales y Contenidos Mínimos Obligatorios para el plan de formación general en la Actualización del Marco Curricular del año 2009 implicaron, en lo específicamente relacionado con el sector de Matemática, que especialmente en el eje de Datos y Azar, hubo contenidos que se eliminaron y otros que ingresaron al plan de formación general, que ahora se enseña en los cuatro niveles de la enseñanza media y con una mayor profundización, cambio que igualmente ha recogido la Prueba de Selección Universitaria, PSU que ha ido incorporando en forma progresiva en la PSU de Matemática los contenidos de esta actualización, hasta la admisión 2017, de modo de que los establecimientos educacionales realicen una real implementación de esta actualización curricular a nivel nacional (DEMRE, 2015).

Si se considera que el léxico disponible se refiere al número de palabras que se presentan a la mente del hablante, en este caso los estudiantes, de forma inmediata y natural cuando se trata de un determinado tema, es razonable pensar que los estudiantes de la carrera de Pedagogía en Matemática que pueden referir un mayor número de palabras en este dominio tienden a tener mejores promedios de calificaciones que sus pares que logran referir un menor número de ellas. Esta evidencia es congruente con otros hallazgos que no sólo muestran un desarrollo del léxico insuficiente en los estudiantes, sino que las mismas debilitan sus estructuras semánticas necesarias para alcanzar de forma óptima el aprendizaje en matemáticas (Ferreira et al., 2014). En Matemáticas la inadecuada comprensión de conceptos matemáticos o su confusión probablemente es un obstáculo relevante a la hora de resolver problemas, expresado en el 
empleo incorrecto de propiedades y definiciones algebraicas; interpretación incorrecta del lenguaje; errores al operar algebraicamente o datos mal utilizados. Asumiendo que los errores y dificultades encontradas por los estudiantes al resolver este tipo de problemas no se debe a una insuficiente ejercitación, sino más parece tener su génesis en un inadecuado uso y énfasis de los aspectos conceptuales ligados al uso de letras, propiedades y conceptos, como también, a la falta de una conexión entre éstos con las propiedades de las operaciones numéricas.

Estas debilidades en el léxico disponible del estudiante, que repercute en su rendimiento, podría encontrar su explicación en el proceso de enseñanza-aprendizaje recibido en matemáticas por el estudiante a lo largo de su itinerario escolar, pues en el espacio escolar los estudiantes raras veces reciben retroalimentación de parte de sus profesores cuando dan respuestas erróneas, y tampoco reciben de parte de ellos preguntas que propicien que ellos mismos se percaten o constaten el tipo y naturaleza de sus errores (Rach et al., 2013). Del mismo modo, los estudiantes chilenos si bien logran realizar aplicaciones simples, como problemas de operatoria sencilla y rutinarios, presentan serias dificultades para resolver problemas que exigen razonamientos analíticos y mecanismos de evaluación y tampoco son capaces de realizar aplicaciones a las situaciones cotidianas a partir de la Matemática (MINEDUC 2010). Todas estas situaciones desfavorables al aprendizaje de las matemáticas se incrementan, si se observa las interacciones de los profesores de matemáticas en las aulas de clase en Chile, las cuales se focalizan mayoritariamente en la presentación mecánica de información y la resolución mecánica de problemas (Radovic y Preiss, 2010; Preiss et al, 2011), En lo anterior juegan un rol relevante el tipo de metodología del profesor. Su modelaje y expectativas respecto del aprendizaje de sus estudiantes, los recursos y tipos de tareas utilizados pueden ir generando paulatinamente sentimientos de incapacidad, ansiedad, aversión o inatención que impactan desfavorablemente en el logro de aprendizajes de sus estudiantes (Vandecandelaere et al, 2012; Yaratan y Kasapoglu, 2012). Esta relación estrecha entre las prácticas de modelado y las actitudes que manifiestan los estudiantes hacia las matemáticas se constata en aquellos estudiantes de procesos de formación inicial en futuros docentes (Bilgic y Uzel, 2014).

Sobre la base de estas evidencias, es posible sugerir que las autoridades y agentes involucrados en los procesos de formación deben profundizar sus acciones tendientes a fortalecer la comprensión de los conceptos matemáticos que fortalezcan la apropiación y uso del léxico disponible a la hora de acometer la resolución de tareas y problemas de las diversas asignaturas de su plan de formación. Adicionalmente, en el ámbito de la formación pedagógica, se debería fortalecer el desarrollo del razonamiento formal, es decir, fomentar que él(la) estudiante pueda razonar sin que esté presente aquello sobre lo cual razona. Esta evidencia permite sostener que los futuros profesores en sus procesos de formación deben recibir enfoques y herramientas didácticas encaminadas a fortalecer o desarrollar un enriquecimiento del lexicón asociado a estos dominios. Para aprender matemática se necesita compartir un léxico general, disponible, y un léxico específico, que debe ser incorporado por la persona que aprende y añadido al léxico general disponible.

La resolución de problemas que deben enfrentar los estudiantes de Pedagogía en Matemáticas exige de parte de ellos poner en juego diversas habilidades, como abstraer, probar, argumentar y encontrar el sentido de las ideas y conceptos matemáticos que subyacen a la misma. En esta perspectiva, un problema es una herramienta que permite pensar matemáticamente, y a la vez, exponer y auscultar los diversos esquemas de resolución implicados en el mismo, en la medida en que los estudiantes en su rol de resolutores deben ser capaces de preguntarse por los hechos, las interpretaciones y las explicaciones necesarias para abordar su solución. De ahí la relevancia de identificar el nivel de desarrollo de estos esquemas de razonamiento en los estudiantes de Pedagogía en Matemática.

Por lo mismo, los profesores encargados de los procesos de formación deben proveer a los futuros maestros, y a su vez, éstos deben interiorizar como futuros profesores de matemáticas, la necesidad de distinguir entre la respuesta que sus estudiantes dan a un problema y los procedimientos o estrategias que emplean en dicho proceso, y lo que resulta mayormente relevante es esto último, es decir los métodos y estrategias puestos en juego. El proceso y los procedimientos involucrados en la resolución de problemas y el razonamiento matemático, constituyen el elemento central del currículum en matemática chileno y a nivel internacional (MINEDUC, 2009; NRC, 2009). Finalmente, se puede sostener que junto con la eventual reformulación y mejora de los procesos de formación inicial de los docentes acogiendo estas evidencias, existe la posibilidad cierta de perfeccionar a los profesores en ejercicio, especialmente aquellos que ejercen en la enseñanza básica sin mención, con la finalidad de mejorar sus prácticas pedagógicas, tal como lo ponen en evidencia programas pilotos en ésta línea, que perseguían que los profesores en ejercicio identificaran patrones a través de la preparación de sus clases y de la didáctica utilizada (Díaz y Poblete, 2009).

Proveer a los estudiantes de Pedagogía en Matemática, futuros profesores en formación, de entrenamiento intracurricular de estrategias de disposición al estudio y su posterior uso, podrían contribuir no sólo a potenciar el desarrollo de esquemas de razonamiento lógico formal y una adecuada conceptualización 
disciplinar aumentando su léxico disponible, sino también a una mejor integración y disposición motivacional de las nuevas generaciones de universitarios a las instituciones de educación superior (Broc, 2011; ElviraValdés y Pujol, 2014). Este tipo de acciones de apoyo redundarán en modificar creencias, procesos de autorregulación del aprendizaje, manejo del tiempo y estilos de atribución de los estudiantes respecto del esfuerzo emprendido y del resultado alcanzado en sus demandas académicas como en su eventual fracaso o adaptación a la vida universitaria (Fernández et al., 2015; Soares et al., Guisandes, 2011).

De acuerdo al primer modelo multivariado que se analizó, existen dos factores propios del proceso formativo de los futuros profesores de matemáticas que tienen directa incidencia en su rendimiento académico: la disponibilidad léxica en el ámbito de datos y azar y los esquemas de razonamiento formal (modelo 3, con las 15 variables originales analizadas). Este hallazgo es relevante, por cuanto ambos factores de tipo cognitivo pueden ser adecuadamente considerados y potenciados en las mallas formativas, por sobre aquella variable de naturaleza estructural como lo es la universidad a la cual asisten. El hecho que figure como relevante el centro de interés de datos y azar podría ser justificado dado que el trabajo en este dominio de contenido involucra un nivel de abstracción que obliga al alumno al uso de habilidades cognitivas superiores, como interpretar, inferir, representar, describir, etc.

Del segundo modelo multivariado en el que se analizan sólo factores de tipo no estructurales, se desprende que la disponibilidad léxica asociada a los centros de interés matemáticos constituyen factores relevantes al momento de explicar la variabilidad del rendimiento académico de los futuros profesores de matemáticas (modelo 4, Tabla 4),. De este modo los ejes de geometría, datos y azar y sistemas numéricos, se erigen como tres de las cuatro variables que formar parte del modelo reportado. Esto puede deberse al hecho que en general, en las asignaturas relacionadas al dominio disciplinar se requiere comunicar, interpretar, organizar y aplicar conceptos matemáticos fundamentales, junto al ejercicio habilidades cognitivas superiores inherentes a la resolución de tareas y problemas en cada una de las asignaturas de su malla de formación.

En particular, la preeminencia de tópicos asociados a la disponibilidad léxica en centro de interés de datos y azar en prácticamente todos los modelos considerados, se ve corroborada por el hecho que emerge de forma conjunta la variable esquemas de razonamiento formal asociadas a la resolución de problemas de probabilidades en el segundo modelo propuesto. Esta confluencia puede encontrar su explicación en que la adecuada comprensión de conceptos relativos al azar requiere por parte del estudiante de pedagogía de un nivel alto de desarrollo que le permita intuir, comprender y cuantificar la incertidumbre asociada a las situaciones de carácter aleatorio, en las cuales están implicadas las habilidades de identificar, organizar y jerarquizar adecuadamente la información relevante.

El primer modelo da cuenta de diferencias significativas respecto de las instituciones de pertenencia de los estudiantes que fueron parte del estudio. Esto puede estar sugiriendo cierto tipo de "prestigio institucional" que incide en la preferencia de los estudiantes al momento de postular a las formaciones en pedagogía. En efecto, si ambas instituciones están asociadas a sectores socioeconómicos relativamente homogéneos, están localizadas en una misma localidad geográfica, y paralelamente, y si se considera la existencia de la llamada "Beca Vocación de Profesor", que permite realizar los estudios de pedagogía gratuitamente a todos aquellos estudiantes que ponderan sobre un puntaje específico de admisión en la Prueba de Selección Universitaria nacional, la elección de su formación en pedagogía por consideraciones económicas no parece ser sostenible. Por lo anterior, es posible inferir que el mecanismo plausible para definir la opción de ingreso de los estudiantes debiera ser realizado por criterios de prestigio o tradición, información pública de ranking de las instituciones, información sobre acreditación de las carreras, etc.

El estudio cuenta con limitaciones que se han de considerar a la hora de analizar sus principales hallazgos. Uno de los principales que pueden ser relevados es el hecho que la variable observada rendimiento académico de los estudiantes es determinada como un promedio de calificaciones, las cuales han sido asignadas por los docentes responsables de las asignaturas de las diversas mallas de formación de las instituciones universitarias analizadas, y por lo mismo, pueden tener un sesgo asociado, entre otros, a los criterios de evaluación, tipos, frecuencia y ponderación de evaluaciones, niveles de exigencia de los mismos agentes educativos. Del mismo modo, pueden existir potenciales sesgos asociados a diferencias de enfoques y énfasis curriculares o de metodologías de enseñanza, las cuales podrían contribuir de forma importante en la variabilidad de los resultados. Se espera poder trabajar en el futuro con un diseño de investigación que permita controlar o estimar el efecto de estos factores.

\section{CONCLUSIONES}

1.- Existen dos factores propios del proceso formativo de los futuros profesores de matemáticas que tienen directa incidencia en su rendimiento académico: la disponibilidad léxica en el ámbito de datos y azar y los esquemas de razonamiento formal. 
2.- La disponibilidad léxica asociada a los centros de interés matemáticos constituyen factores relevantes al momento de explicar la variabilidad del rendimiento académico de los futuros profesores de matemáticas.

3.- La preeminencia de tópicos asociados a la disponibilidad léxica en centro de interés de datos y azar en prácticamente todos los modelos considerados, se ve corroborada por el hecho que emerge de forma conjunta la variable esquemas de razonamiento formal asociadas a la resolución de problemas de probabilidades en el segundo modelo propuesto.

4.- El primer modelo da cuenta de diferencias significativas respecto de las instituciones de pertenencia de los estudiantes que fueron parte del estudio. Esto sugiere cierto tipo de "prestigio institucional" que incide en la preferencia de los estudiantes al momento de postular a las formaciones en pedagogía.

5.- El estudio cuenta con limitaciones que se han de considerar a la hora de analizar sus principales hallazgos: (i) la variable observada rendimiento académico de los estudiantes es determinada como un promedio de calificaciones lo que puede tener un sesgo asociado; y (i) pueden existir potenciales sesgos asociados a diferencias de enfoques y énfasis curriculares o de metodologías de enseñanza

\section{AGRADECIMIENTOS}

Trabajo apoyado por el proyecto Fondecyt Regular N $N^{\circ} 1140457$ Plataforma adaptativa On line para el fortalecimiento de las competencias matemáticas y pedagógicas a partir del estudio léxico semántico de estudiantes y profesores de pedagogía en matemática, y por el Proyecto Basal FB0003 del Programa de Investigación Asociativa de Conicyt. La investigación contó con el apoyo de los tesistas señores, Juan Vergara, Alex Barriga, Felipe Cortés y Diego Silva

\section{REFERENCIAS}

Bilgic, E.N., Uzel, D. The Attitudes of the Elementary School Mathematics Teacher Candidates towards Proof in the Mathematical Modelling Proccess. DOI:10.1016/j.sbspro.2014.01.349 Social and Behavioral Sciences (en línea). 116, 1083-1087 (2014)

Broc, M. Voluntad para estudiar, regulación del esfuerzo, gestión eficaz del tiempo y rendimiento académico en alumnos universitarios. Revista de Investigación Educativa, 29(1), 171-185 (2011)

Casanova, D. Equidad de acceso a la educación superior: El "puntaje ranking de notas" como mecanismo de inclusión en el sistema de admisión de Chile. DOl:1014507/epaa.v23.1908. Archivos Analíticos de Políticas Educativas (en línea), 23(72) (2015)

Centro de Microdatos de la Universidad de Chile. Informe final estudio sobre causas de la deserción universitaria. http://www.opech.cl/educsuperior/politica_acceso/informe_final_causas_desercion_universitari a.pdf (2008) Recuperado el 21 de mayo de 2015

Cerda, G., Ortega, R. y otros 3 autores. Inteligencia lógica y extracción social en estudiantes talentosos y normales de enseñanza básica y media en Chile. Anales de Psicología, 27(2), 389-398 (2011)

Cerda, G., Pérez, C. y Melipillán, R. Test de Inteligencia Lógica Superior (TILS). Manual de Aplicación. Concepción:Universidad de Concepción. 55 páginas (2010)

DEMRE. Temario de la Prueba de Matemática. Departamento de Evaluación, Medición y Registro Educacional, Universidad de Chile (2015)

Díaz, V. y Poblete, A. Perfeccionamiento en matemáticas basado en competencias para docentes de escuelas básicas municipalizadas de la región de Los Lagos y de Los Ríos. DOI:10.4067/S071807052009000200001. Estudios Pedagógicos (en línea), 35(2), 13-34 (2009)

Donoso, S. y Schiefelbein, E. Análisis de los modelos explicativos de retención de estudiantes en la universidad: una visión desde la desigualdad social. DOI: 10.4067/S0718-07052007000100001. Estudios Pedagógicos (en línea), 33(1), 7-27 (2007)

Elvira-Valdés, M. y Pujol, L. Variables cognitivas e ingreso universitario: predictores del rendimiento académico. Universitas Psychologica, 13(4), 1557-1567 (2014)

Fernández, A., Arnaiz, P., Mejía, R. y Barca, A. Atribuciones causales del alumnado universitario de República Dominicana con alto y bajo rendimiento académico. DOI: 10.17979/reipe.2015.2.1.1319. Revista de Estudios e Investigación en Psicología y Educación (en línea), 2(1), 19-29 (2015)

Ferreira, A.; Salcedo, P.; del Valle Estudio de disponibilidad léxica en el ámbito de las matemáticas. Estudios Filológicos, 54, 69-84 (2014) 
García, M. La autorregulación académica como variable explicativa de los procesos de aprendizaje universitario. Revista de Currículum y Formación del Profesorado, 16(1), 203-221 (2012)

Jones, M. G., Gardner, G., Taylor, A. R., Wiebe, E., y Forrester, J. Conceptualizing magnification and scale: The roles of spatial visualization and logical thinking. DOI: 10.1007/s11165-010-9169-2. Research in Science Education (en línea), 41(3), 357-368 (2011)

Kontons, D., Van Gog, T., Paas, F. Training self-assessment and task-selection skills: A cognitive approach to improving self-regulated learning. DOI: 10.1016/j.learninstruc.2011.08.004.bLearning and Instruction (en línea), 22(2), 121-132 (2012)

Leese, M. Bridging the gap: Supporting student transitions into higher education. Journal of Further and Higher Education, 34 (2), 239-51. (2010)

Maris, S. y Difabio, H. Logro académico y pensamiento formal en estudiantes de ingeniería. Electronic Journal of Research in Educational Psychology, 7(2), 653-672 (2009)

MINEDUC, Análisis de las competencias en NB1: caracterización de los niveles de complejidad de las tareas matemáticas: Santiago Chile (2009)

MINEDUC Resumen de Resultados PISA 2009 Chile. Ministerio de Educación, Unidad de Curriculum y Evaluación (2010)

MINEDUC. Deserción en la educación superior en Chile. Serie Evidencias En línea] Año 1(9), Recuperado el 21 de abril de 2015 de http:// centroestudios.mineduc.cl (2012a)

MINEDUC Resultados Evaluación Inicia. Recuperado el 07 de abril de 2015 de http://www.mineduc.cl/usuarios/mineduc/doc/201308221629100.RESULTADOS_EVALUACI ON_INICIA.pdf. (2012b)

MINEDUC Guías Didácticas para la Articulación de los Ejes Curriculares de Números, Álgebra, Geometría Asignatura: Matemática 10 a 40 año de Educación Media. (2012c).

http://portales.mineduc.cl/usuarios/media/doc/201305091223340.Ejes_numero_algebra_geometria.pdf

National Research Council. Mathematics learning in early childhood: Paths towards excellence and equity. Washington, DC: The National Academies Press (2009)

National Council of Teachers of Mathematics. Principles and Standards for School Mathematics. Reston, VA: National Council of Teachers of Mathematics (2000)

Pérez, M., Valenzuela, M., Díaz, A., González-Pienda, J., y Núñez, J. Dificultades de aprendizaje en estudiantes universitarios de primer año. doi.org/10.4067/S0718-04622013000200010. Atenea (en línea), 508, 135-150 (2013)

Preiss, D., Larraín, A., y Valenzuela, S. Discurso y Pensamiento en el Aula Matemática Chilena. Discourse and Thought in the Chilean Mathematics Classroom. Psykhe, 20(2), 131-146 (2011)

Rach, S., Ufer, S., y Heinze, A. Learning from Errors: Effects of Teachers Training on Students' Attitudes towards and their Individual Use of Errors. PNA 8(1), 21-30 (2013)

Radovic, D., y Preiss, D. Patrones de Discurso Observados en el Aula de Matemática. Psykhe, 19(2), 65-79 (2010)

Romero, M. y Pérez, M. Cómo motivar a aprender en la universidad: una estrategia fundamental contra el fracaso académico en los nuevos modelos educativos. Rev. Iberoam. de Educación. 51, 87-105. (2009)

Salazar, J. M. y Leihy, P. S. El Manual Invisible: Tres décadas de políticas de educación superior en Chile (1980-2010). Archivos Analíticos de Políticas Educativas, 21(34). Recuperado [22 de enero de 2016] http://epaa.asu.edu/ojs/article/view/1127 (2013).

Salcedo, P.; Del Valle, M.;Pinninghoff, M.; y Contreras, R. LEXMATH - A Tool for the Study of Available Lexicon in Mathematics. DOI: 10.1007/978-3-319-18833-1_2. Lecture Notes in Computer Science (en línea), 9108 (2), 11-19 (2015)

Salcedo, P.; Ferreira, A., y Barrientos, F. A Bayesian model for lexical availability of Chilean High School students in Mathematics. Lecture Notes in Computer Science, Natural and Artificial Models in Computation and Biology, 7930 (1), 245-253 (2013)

Sanford, T., Hunter, J. M. Impact of Performance-funding on Retention and Graduation Rates Education Policy Analysis Archives, 19(33). Recuperado (22 de enero 2016] desde http://epaa.asu.edu/ojs/article/view/949 (2011) 
Sistema de Información de la Educación Superior, SIES, El aseguramiento de la calidad de la educación superior en Chile. [En línea]. Santiago, (2012)

Soares, A., Almeida, L., y Guisandez, A. Ambiente académico y adaptación a la universidad: un estudio con estudiantes de $1^{\circ}$ año de la universidad Do Minho. Rev. Iberoam. de Psicología y Salud, 2(1), 99-121 (2011)

Tatto, M. T., Schwille, J., Senk, S. L., Ingvarson, L., Rowley, G., Peck, R.,Reckase, M. Policy, Practice, and Readiness to Teach Primary and Secondary Mathematics in 17 countries. Findings from the IEA Teacher Education and Development Study in Mathematics (TEDS-M). Amsterdam: IEA. (2012)

Tobin, K., y Capie, W.. The development and validation of a group Test o Logical Thinking. Educational and Psychological Research and Evaluation, 41(2), 413-419 (1981)

Tuckman, B., y Kennedy, G. Teaching learning strategies to increase success of first-term college students. DOI:10.1080/00220973.2010.512318. The Journal of Experimental Education, 79(4), 478-504. (2011)

Urzúa, P., Sáez, K., y Echeverría, M. Disponibilidad Léxica Matemática. Análisis cuantitativo y cualitativo. Revista de Lingüística Teórica y Aplicada Concepción, 44(2), 59-76 (2006)

Vandecandelaere, M., Speybroeck, S., Vanlaar, G., De Fraine, B., y Van Damme, J. Learning environment and students' mathematics attitude. DOI:10.1016/j.stueduc.2012.09.001. Studies in Educational Evaluation (en línea), 38, 107-120 (2012)

Yaratan, H., y Kasapoglu, L. Eighth grade students' attitude, anxiety, and achievement pertaining to mathematics lessons. DOI: 10.1016/j.sbspro.2012.05.087. Social and Behavioral Sciences, 46, 162-171 (2012)

Yau, H., Sun, H., y Cheng, A. Adjusting to university: the Hong Kong experience. DOI: 10.1080/1360080X.2012.642328. Journal of Higher Education Policy and Management (en línea), 34(1), 15$27(2012)$ 
